\title{
A Case Study on Asprox Infection Dynamics
}

\section{Youngsang Shin', Steven Myers², Minaxi Gupta'}

I School of Informatics and Computing, Indiana University \{shiny,minaxi\}@cs.indiana.edu

${ }^{2}$ School of Informatics and Computing, Indiana University samyers@indiana.edu 


\section{Asprox Overview}

- Brief History

- Asprox botnet has been around since 2007

- Initially used exclusively for sending phishing emails

- Around May 2008, a new update was pushed to Asprox bots

> an attempt to grow the size of the botnet

> SQL injection vector

- A significant number of web servers have since been attacked and their unsuspecting visitor machines turned into Asprox bots 


\section{Multistep Life Cycle of Asprox}

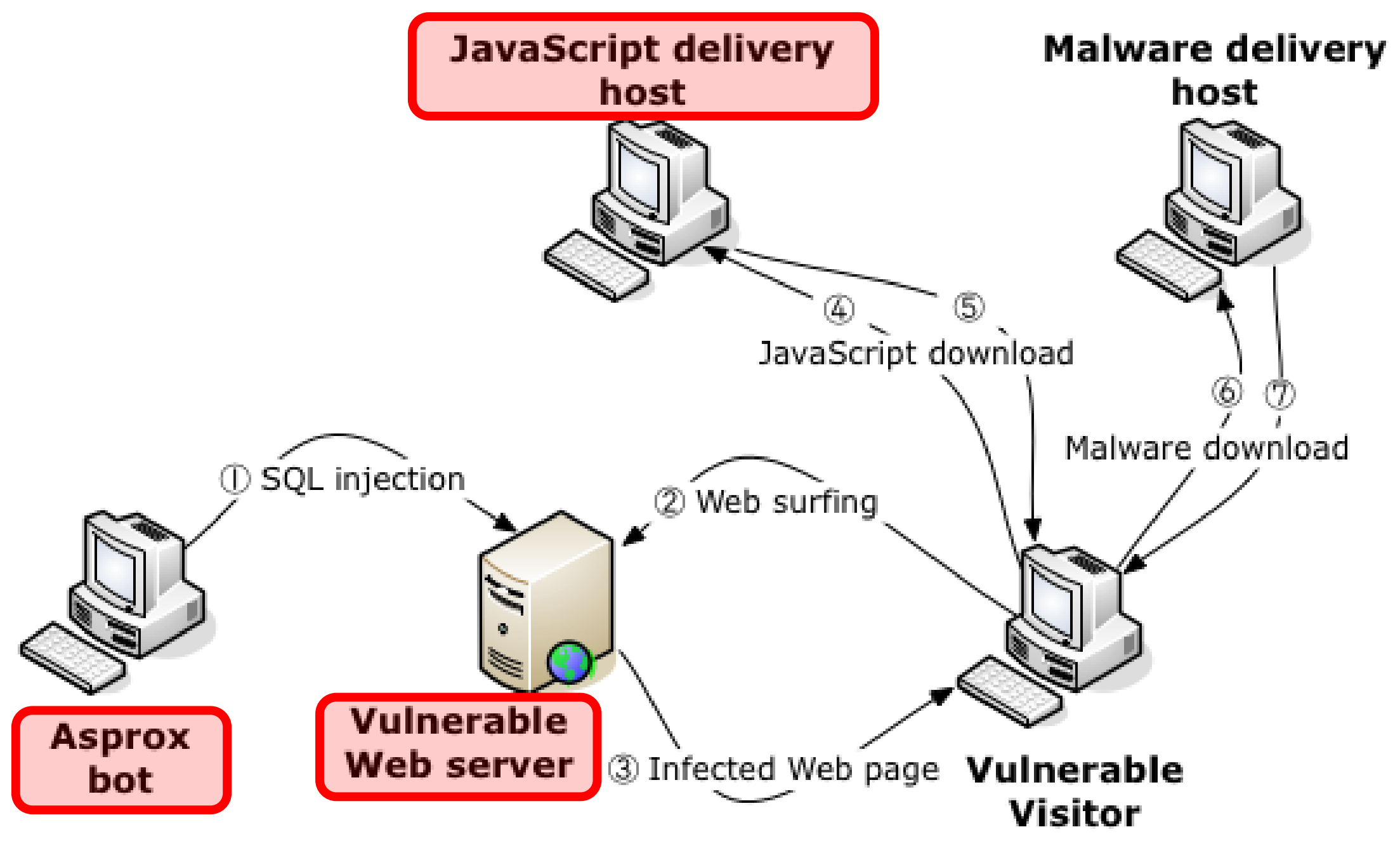




\section{Outline}

- Introduction

- Data Collection \& Overview

- Analysis of Asprox Infection Dynamics

- Asprox Bots

- Infected Web Servers

- JavaScript-Delivery Hosts

- Concluding Remarks 


\section{Data on SQL-injecting Asprox Bots}

- Information about Asprox bots that attacked web servers at Indiana University in August 2008

- SQL-injection attacks

Collection Period

Unique IP addresses of attacking bots

Autonomous systems attackers belonged to

Web servers targeted
8/9/2008 8/25/2008 (I 7 days)

57,419

1,847

581 


\section{Data on JavaScript-Delivery Hosts}

- JavaScript-delivery hosts

Collection Period 10/26/2008 1/3 I/2009 (98 days)

Unique Hostnames 324

With gTLDs 151 (.com: 105, name:28, .mobi:I I, .net:4, .org:3)

With ccTLDs 173 (.ru:I 27, .cn:34, .jp:4, .cc:4, .tk:I, .kz:I, .eu:I, .me:I)

- JavaScript-delivery hosts

- DNS servers for JavaScriptdelivery hosts

\begin{tabular}{|l|l|}
\hline Resolved hostnames & 55 \\
\hline IP addresses & 2,214 \\
\hline ASes & 308 \\
\hline BGP prefixes & 898 \\
\hline Countries & 64 \\
\hline
\end{tabular}

\begin{tabular}{|l|l|}
\hline Resolved hostnames & 619 \\
\hline IP addresses & 147 \\
\hline ASes & 67 \\
\hline BGP prefixes & 115 \\
\hline Countries & 11 \\
\hline
\end{tabular}




\section{Data on Infected Web Servers (1/2)}

- Data collection

- Searched web pages containing the URLs pointing to the malicious JavaScript delivery hosts

- Used Google and Yahoo search APls

- Examined web pages in search results, including the cached pages

- Web-server classification in the search results

- Infected but unreachable

- Infected, reachable, but undecidable

- Infected, reachable, and identifiable 


\section{Data on Infected Web Servers (2/2)}

- Data collection period

- I I/0 I/2008 0 I/3I/2009 (92 days)

\begin{tabular}{|l|c|c|}
\hline Class & \# of Servers & $\%$ \\
\hline Total \# of infected web servers & 8,926 & $100 \%$ \\
\hline Infected but unreachable & 2,751 & $30.82 \%$ \\
\hline Infected, reachable, but undecidable & 1,141 & $12.78 \%$ \\
\hline Infected, reachable, and identifiable & $\mathbf{5 , 0 3 4}$ & $\mathbf{5 6 . 4 0 \%}$ \\
\hline
\end{tabular}




\section{Outline}

- Introduction

- Data Collection \& Overview

- Analysis of Asprox Infection Dynamics

- Asprox Bots

- JavaScript-Delivery Hosts

- Infected Web Servers

- Concluding Remarks 


\section{Analysis of Asprox Bots}

- The number of attacking bots is lesser on weekdays than weekends

- Artifact of the fact that many bots are residential machines

- New bots are added to the pool as the week progresses, with peaks on Saturdays

- Modest number (up to 3,000) of bots are being reused

- More bots are reused on weekend like the trend of the new bot addition 


\section{Attack Times by Asprox Bots}

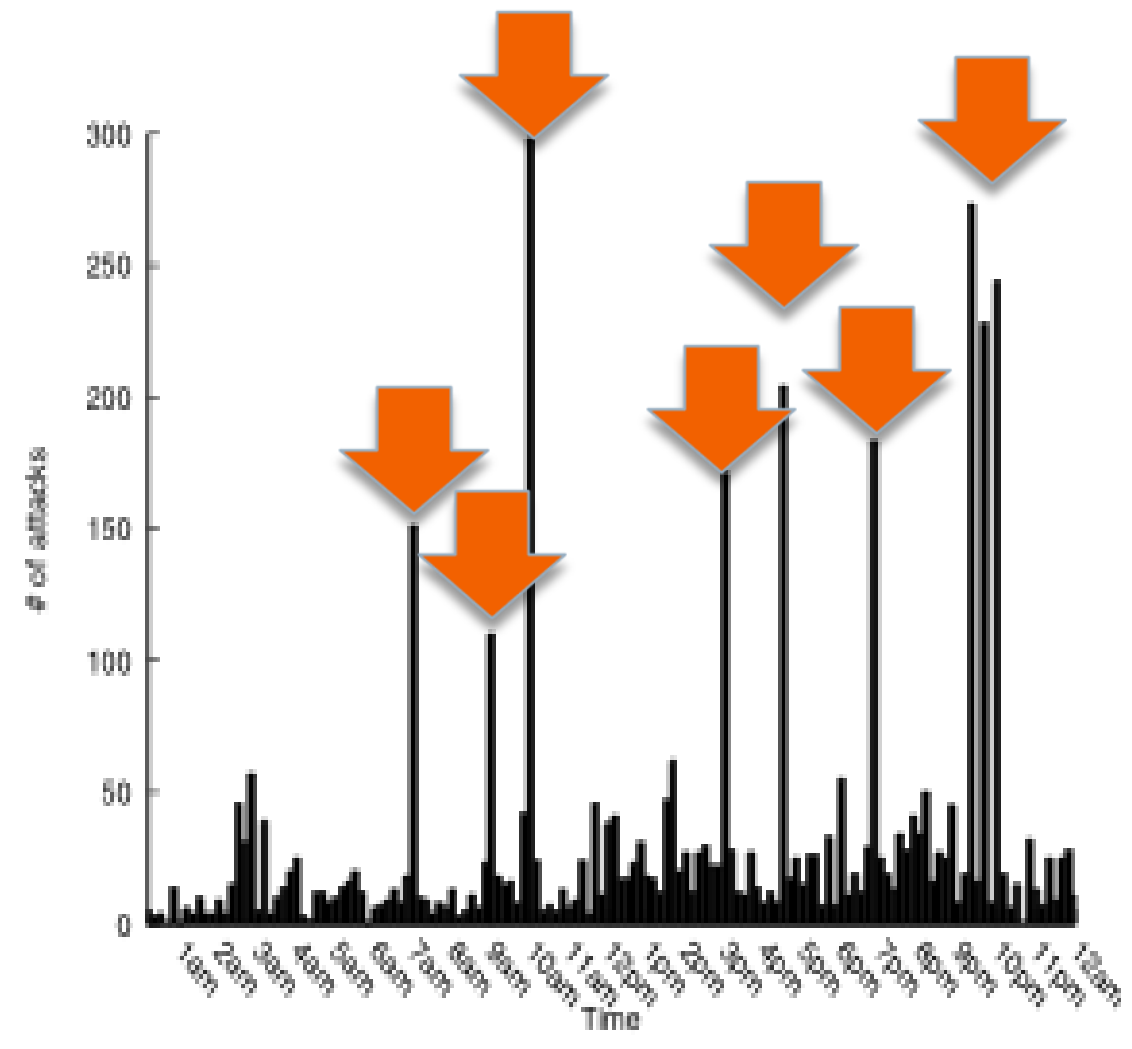

- Asprox bots attacking on a weekend day (8/9)

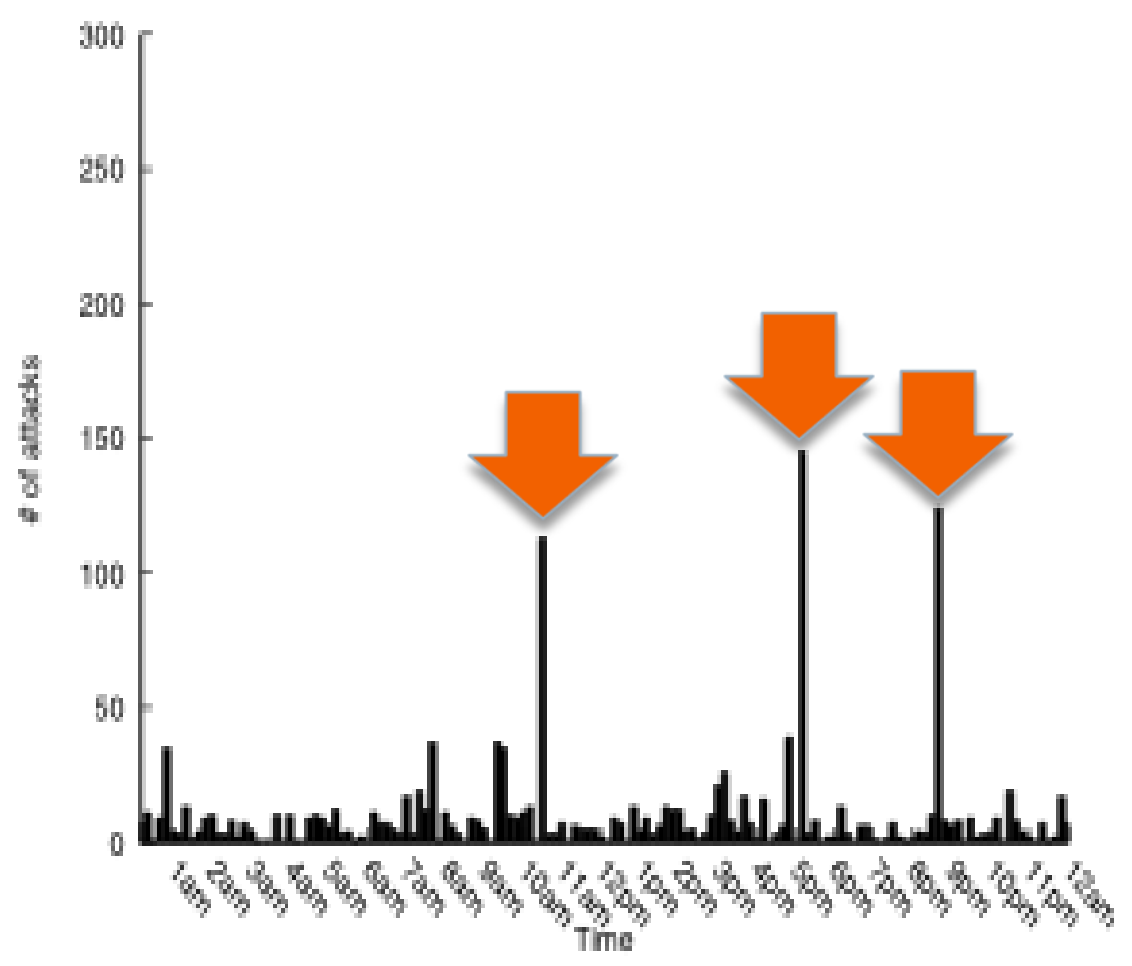

- Asprox bots attacking on a weekday $(8 / 20)$ 


\section{Active Lifetime and Repeated Attacks}

- Around $95 \%$ of attacking bots were observed for less than 2 days

- Helps avoid any IP blacklisting

- Over $50 \%$ of web servers were continuously attacked for 8 days

- $90 \%$ of the bots attacked the same web server about 10 times

- In some cases, one attacker hit the same target over 500 times 


\section{Geographical Distribution of Asprox Bots}

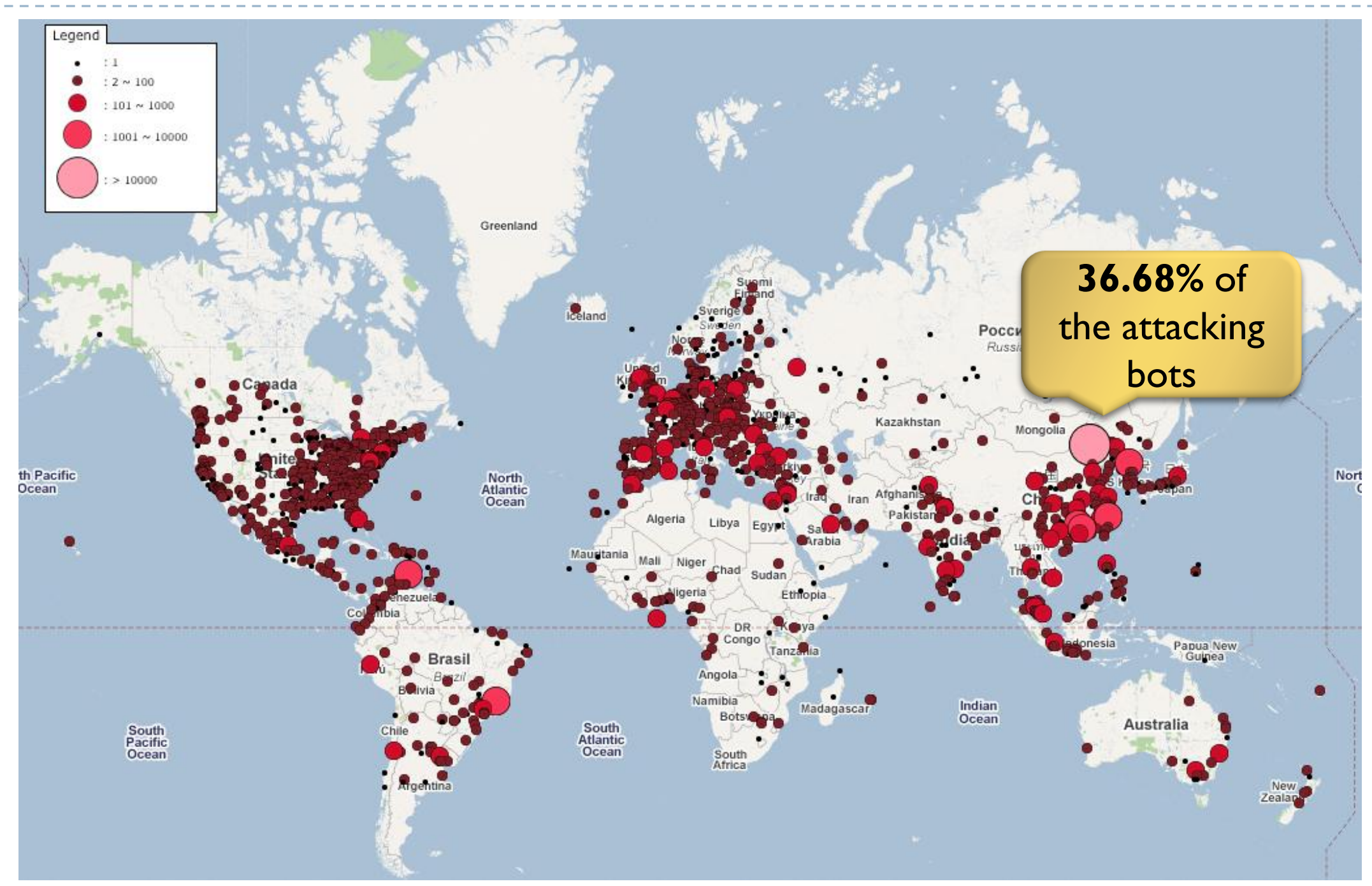




\section{JavaScript-Delivery Hosts}

- Only 27 out of 55 JavaScript delivery hosts were actively used during our data collection period

- Among the 27 JavaScript delivery hosts, $58 \%$ of them appear to be actively fluxing.

- One example, www.berkje.ru

- I,542 IP addresses

- Geographically spread through 60 countries 


\section{\# of IP addresses and IP diversity for}

www . berkje.ru
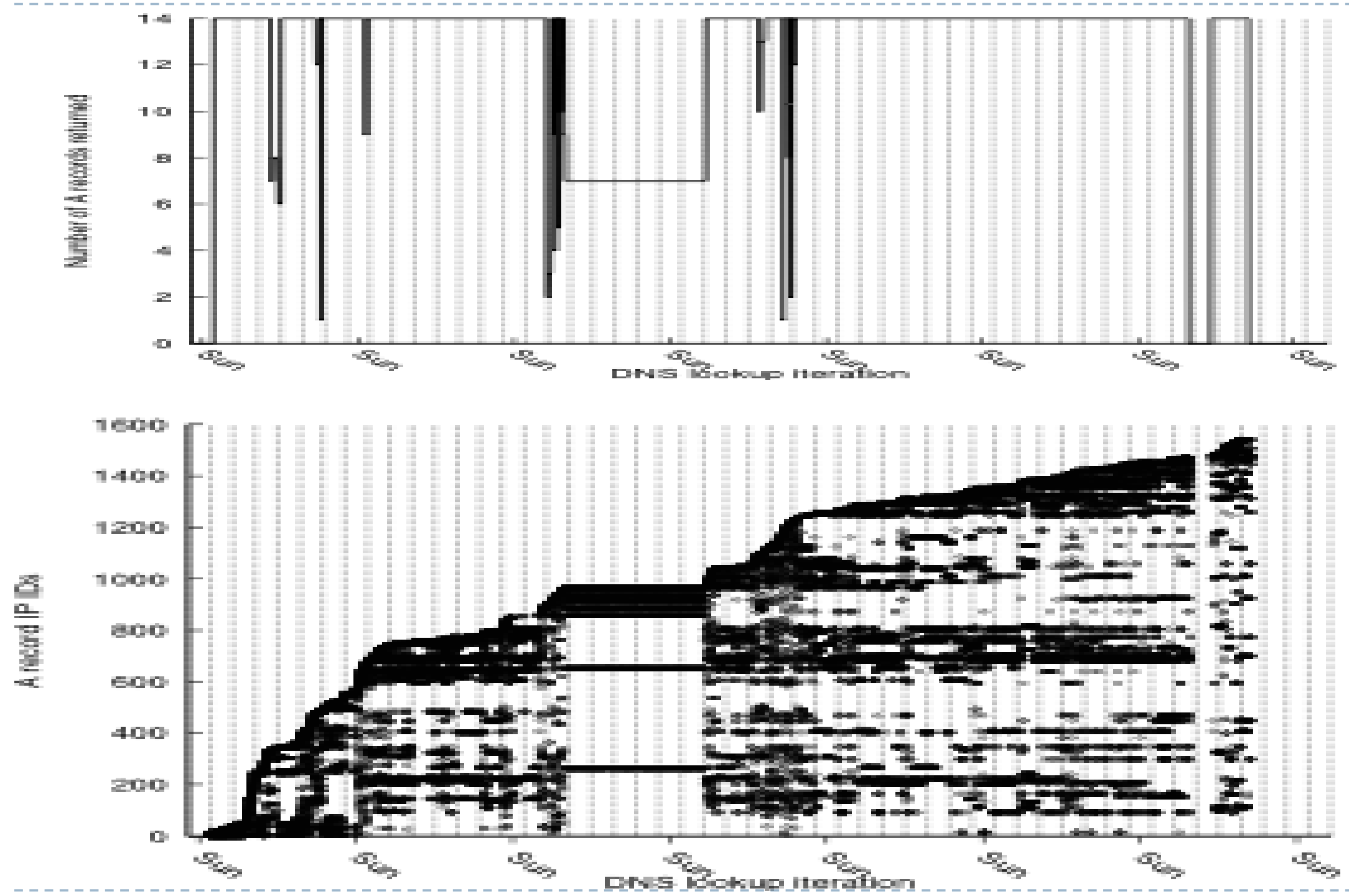

TI INDIANA UNIVERSITY

BLOOMINGTON 


\section{Geo. Dist. of IPs of JavaScript-Delivery Hosts}

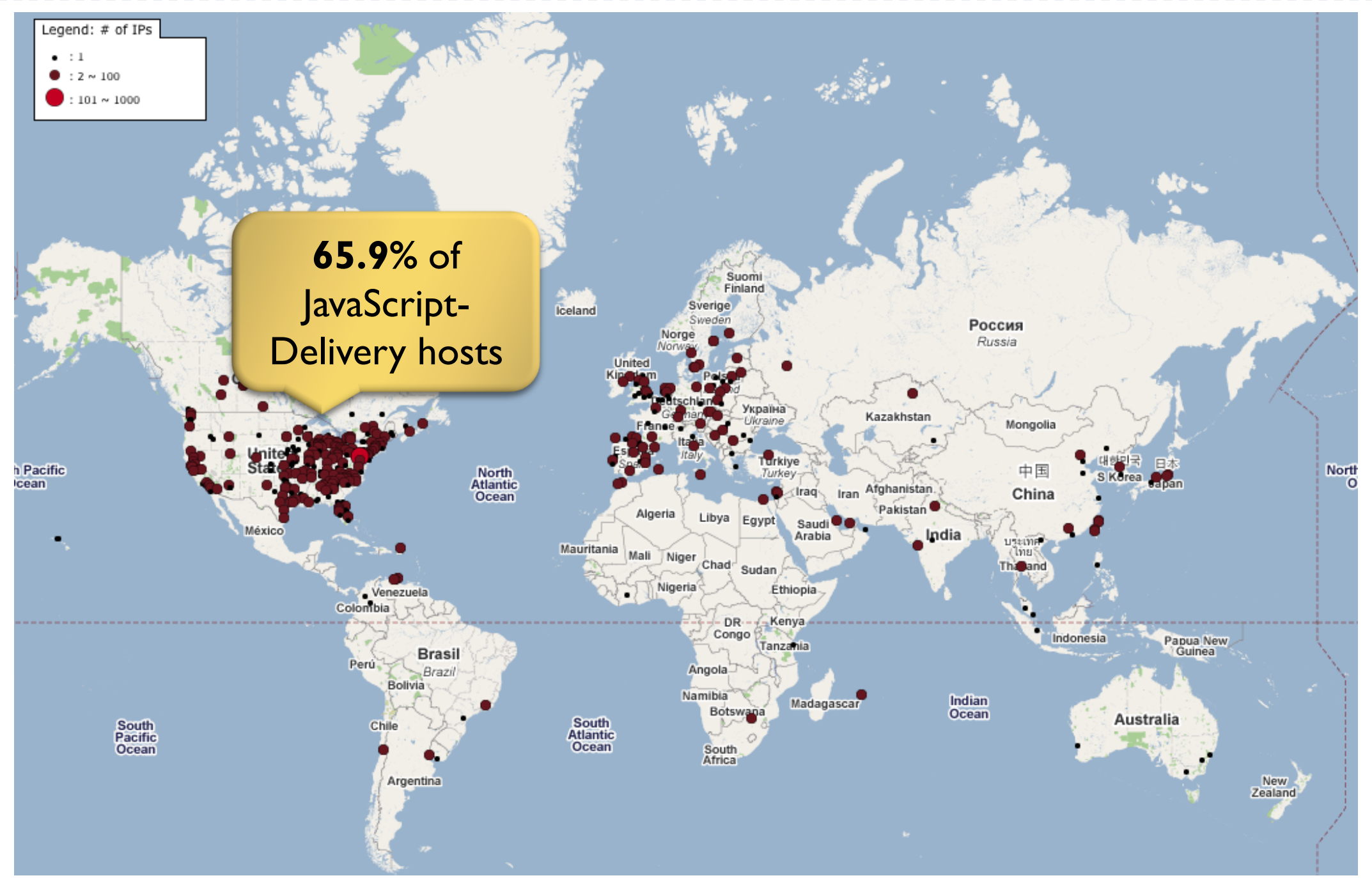




\section{Infected Web Servers}

- TLDs of infected web servers

\begin{tabular}{|l|l|}
\hline TLD & Number of web servers \\
\hline .com & $\mathbf{2 , 3 0 7}$ \\
\hline .pl & 341 \\
\hline .net & 313 \\
\hline .org & 294 \\
\hline .cn & 242 \\
\hline .kr & 201 \\
\hline .uk & 125 \\
\hline Other gTLDs & 105 \\
\hline Other ccTDLs & 1,070 \\
\hline No server name, just IP address & 36 \\
\hline Total Number of web servers & 5,034 \\
\hline
\end{tabular}




\section{Infected Web Servers}

- $\mathbf{7 7} \%$ of the servers were cleaned and the rest stayed infected during our collection period.

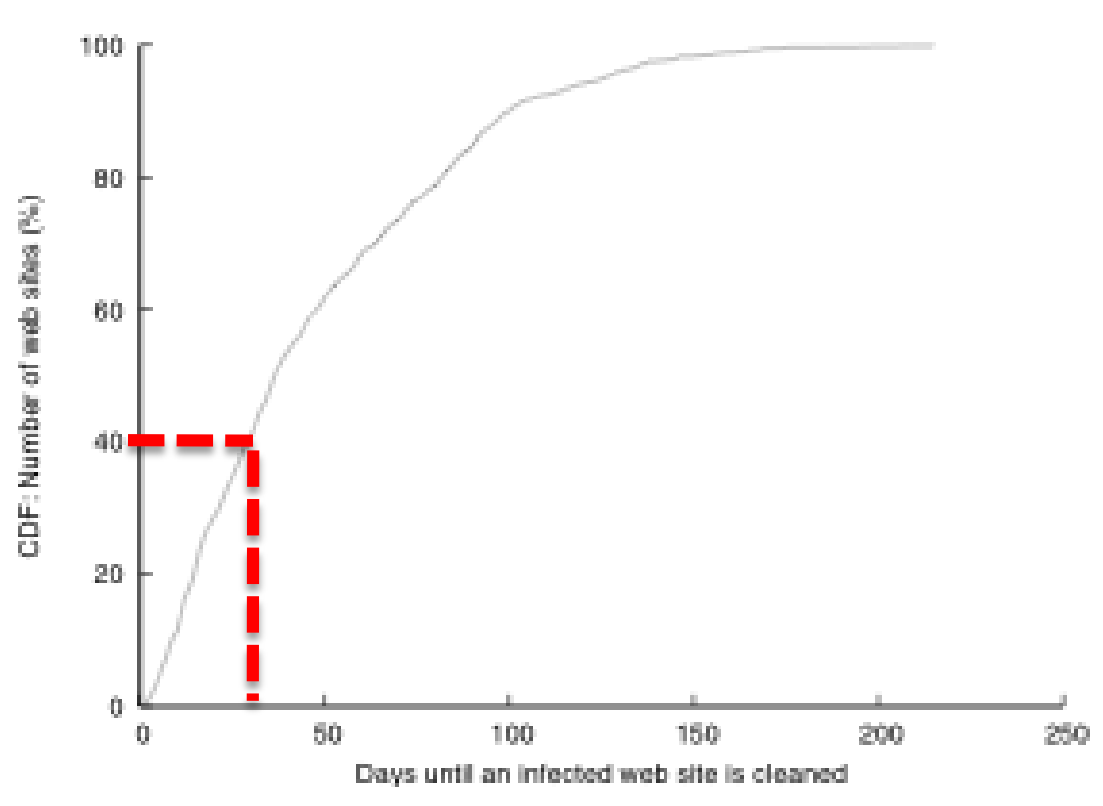

- Cleaned web servers

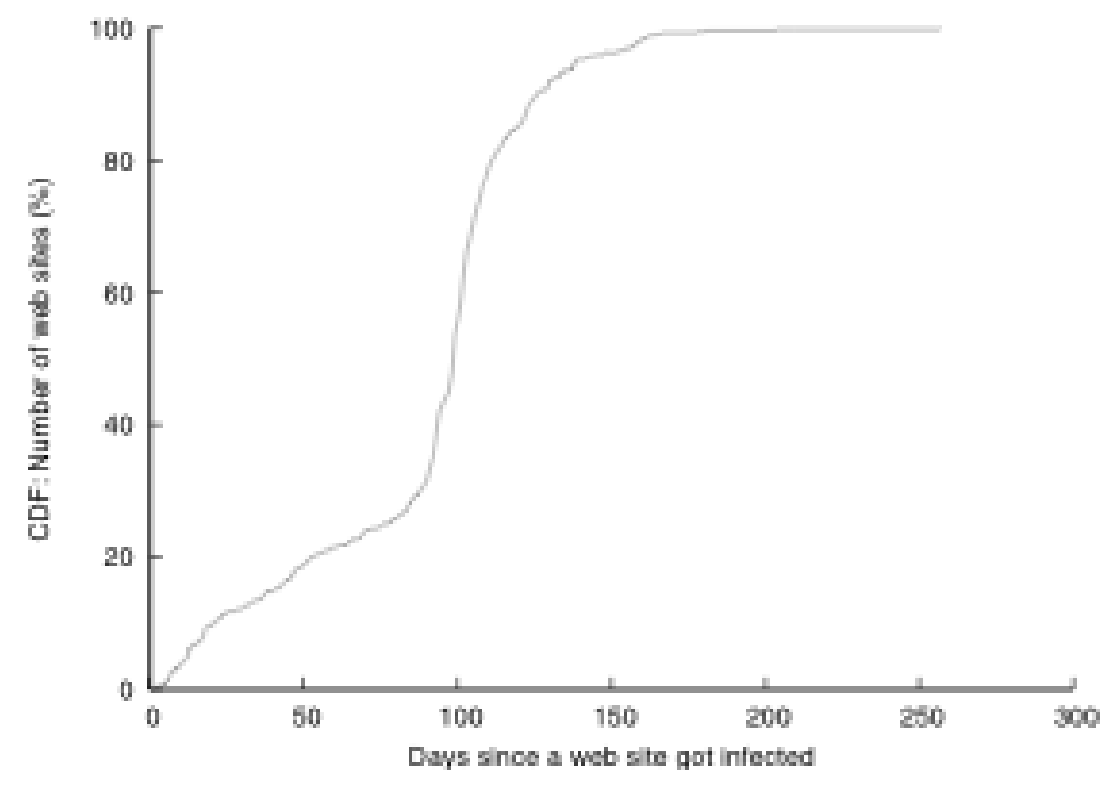

- Still infected web servers 


\section{Conclusion}

- Asprox botnet continues to grow and infect web servers around the world

- Passive monitoring such as Honeypot is not sufficient

v to understand the attack in its entirety or

v to detect changes or modifications to the final vulnerabilities used to attack users' machines or the malware payload delivered

- Adopting the mitigation for the SQL injection attacks would take a long education cycle 


\section{Questions? shiny@cs.indiana.edu}

